\title{
Modified Recursive Least Square Algorithm for THD Reduction using Shunt Active Power Filter in Grid
}

\author{
Divya Prabha \\ M. Tech. Scholar \\ Oriental College of Technology \\ Bhopal, India \\ nehadivya315@gmail.com
}

\author{
Lalit Jain \\ Professor \\ Oriental College of Technology \\ Bhopal, India \\ engg.lalitjain.mlc@gmail.com
}

\begin{abstract}
In industrial and utility power system it has been observed that harmonics build a major role in reducing the quality of power. These harmonics are caused because of increasingly utilize of nonlinear loads connected to the power system. In this context, active power filters play a very significant role for improvement in power quality. Moreover, at transmission ground, there is a high requirement to control reactive power and voltage stability. Conventionally, passive and active filters (shunt and series filters) were used for the development of power quality. But, they suffered from the issues like resonance, fixed compensation, and other heating networks are commonly addressed in the literature as one of the most effective solutions for decreasing the designed.
\end{abstract}

Keyword -active filter, harmonic, hybrid filter.

\section{INTRODUCTION}

Increase in the proliferation of performance-based electronic devices used in electrical devices such as Advanced Speed Drives (ASD), electric arc furnaces, energy saving lamps, uninterruptible power supplies (UPS), power supply switching (SMPS), etc., facilitating the control of devices, robust and flexible. However, these devices contaminate the power system by injecting current harmonics into the $\mathrm{AC}$ grid, providing reactive energy in 3P3W and single-phase systems, producing an unbalance and generating excessive current in the $3 \mathrm{P} 4 \mathrm{~W}$ system. Surveys related to energy quality problems $(\mathrm{PQs})$ have become an important study for utilities and consumers due to the increase in non-linear loads [1]. An in-depth study was conducted to evaluate the problems associated with non-linear load networks. Traditionally, passive LC filters have been used for QP problems.
However, this has significant disadvantages such as large sizing, fixed compensation, resonance effect, etc. Increasing the severity of harmonic loads in the electrical distribution system has attracted the attention and performance of researchers. Engineers implement an effective and robust solution to PQ problems. Customized power devices (CPDs), such as active power filters, are considered an impressive solution to balance the needs of harmonics and reactive power of non-linear loads compared to traditional passive filters.

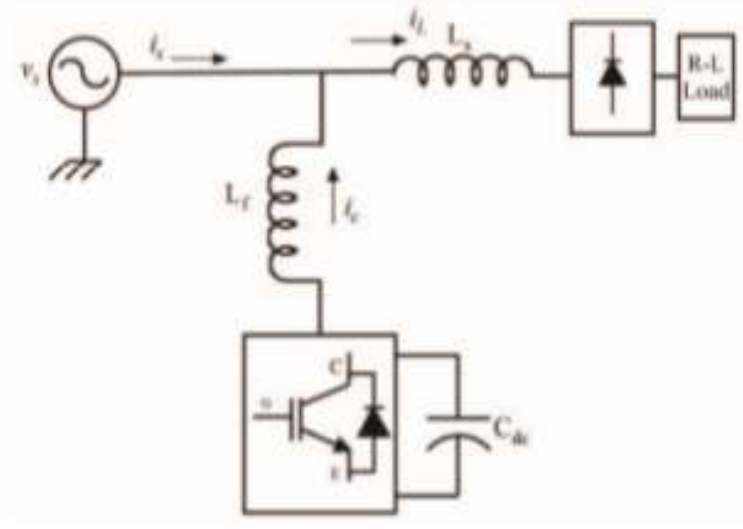

Figure 1 Shunt APF single-line diagram

\section{1) Power System Harmonics}

The harmonics of the network system are integer multiples of the fundamental frequency of the network system. The harmonics of the network are generated by non-linear devices connected to the network. Harmonics are higher frequencies of current and voltage than normal sinusoidal and voltage waveforms. The presence of harmonics (current and voltage) is considered a 
"pollution" that influences the functioning of the power supply systems.

\section{2) Active Power Filters (APF)}

Harmonic distortions in energy distribution systems can be suppressed primarily by active and passive filtering. Passive filtering is the simplest conventional solution to mitigate harmonic distortion. The use of passive elements does not always respond correctly to the dynamics of energy distribution systems. Passive filters are known to cause resonance, thus affecting the stability of energy distribution systems. The frequency variation of the power distribution system and the tolerances on the values of the components influence the characteristics of the passive filter. As regulatory requirements become more stringent, passive filters may not be able to respond to future revisions of a given standard. This may require the introduction of new filters.

\section{LITERATURE REVIEW}

Vijanth S. Asirvadam et al. [1] this paper presents the concept, methodology, and function of minimal recursive LMS and LSD filters (LLS) for real-time harmonic prediction that can be used in process-active filters. Compensate for the compensated delay by obtaining harmonious information. The results show that the RLS and NLMS algorithms work well than LMS and that the mean square error (MSE) generally increases with the increase in the number of harmonic atoms that make up the signal. (C) 2013 the authors. Elsevier Ltd. published technological progress is very challenging nowadays. Non-linear loads in power electronics applications in many applications can lead to power quality problems and one of the main power quality problems is harmonic. Active filters are a commonly used instrument for harmonic attenuation, but the active filter uses digital signal processing (DSP) to calculate harmonic information that causes a delay between the measured and injected harmonics, which can lead to complete cancellation of harmonics.

M. Kelnar et al. [2] This paper presents non-binding computational techniques (combination of fuzzy system and artificial intelligence techniques, also called adaptive neuro-fuzzy interference systems - ANFIS) to design a new control scheme for filter filter switching. active power (SAPF). The predictive and adaptive functions of ANFIS are used to quickly estimate the compensation current. In experiments, a complex adaptive system based on the ANFIS structure was created. The experimental results demonstrate the effectiveness of the proposed control method. This control method reduces harmonics, has a short dynamic response time and effectively improves the overall THD harmonic distortion. It is also useful and applicable to other active filters, especially for non-linear control systems. Compared to other control methods, the simulation and the experimental results show that the proposed control method has the advantages of a shorter reaction time, a good online control and a highly efficient harmonic reduction. .

ChamandeepKaur [3] would ideally have a perfect sinusoidal tension at every point in the network. In reality, it is almost impossible to create such desirable conditions. The currents of voltage and current are strongly different from a sinusoid. These waveform deviations are described by the use of waveform distortion and are commonly referred to as harmonic distortion. Although harmonic distortion is a relatively old phenomenon, it is now one of the main problems for utilities, distribution system operators and their end users. The first disturbances have already occurred during the first years of operation of the energy distribution networks. At that time, the main concern was the effect of harmonic distortions on the electric machines themselves. Another known problem was the interference on telephone lines. In general, however, it can be said that the harmonic distortion previously did not have the same dangerous potential as today. This article presents the design and formulation of hybrid filters to reduce harmonics and improve power quality.

V.K. Arun Shankar et al. [4] This paper examines the modeling of an active shunt power filter (SAPF) based on an integral proportional controller (PI) and a fuzzy logic controller (Fuzzy Logic Controller) for a three wire network to compensate for the current harmonics that generate a high power level to feed non-linear load. In SAPF driven by fuzzy logic, a mathematical model of the system is not required because it is based on a system of inference that contains information derived from human experience. SAPF, based on fuzzy logic and driven by PI, was developed using the MATLAB Simulink Toolkit.

\section{OBJECTIVE}

The work has been done with the following main objectives:

- To design a simple but highly viable hybrid active power buffer that is capable of feeding less distorted voltage to the nonlinear load model.

- To present an optimal controlling of these buffers so as to minimize the voltage distortion by designing a modified RLS algorithm for the same.

- Comparing the THD levels of the output voltage waveform with the standard controlling method with the proposed control design 
- To enhance the system efficiency such that the power inverter produces output with the minimum distortion.

- To further enhance the proposed design such that it is practically feasible to be implemented in grid system having renewable energy resources.

\section{METHODOLOGY}

MATLAB is a high-performance language for technical computing. It integrates computation, visualization, and programming in an easy-to-use environment where problems and solutions are expressed in familiar mathematical notation. Typical uses include: Application development, including Graphical User Interface building.

The modeling has been done in MATLAB/SIMULINK environment. The output results have been analyzed and the control algorithm has been implemented to control the functioning of power inverter model.

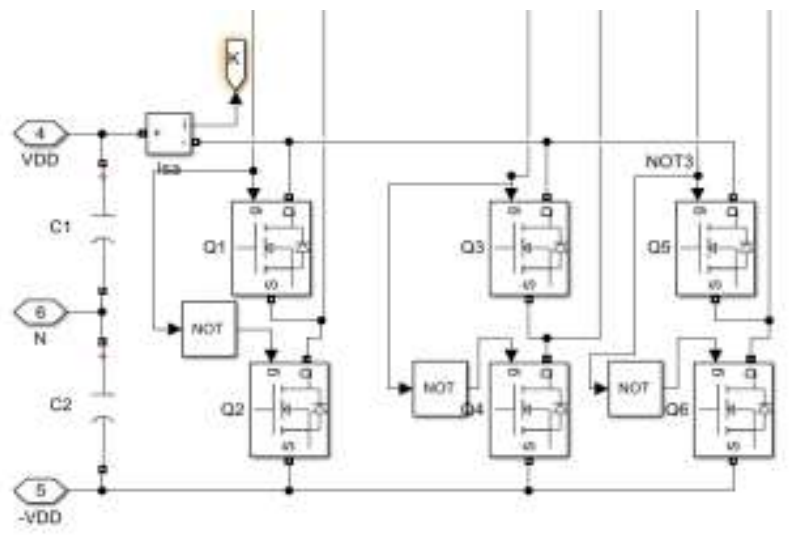

Figure 2 MATLAB model of Designed inverter

\section{A) Instantaneous Power Theory or PQ-Theory}

In this technique, the three-phase voltage and load currents are transformed into the $\alpha-\beta$ frame and with this the $p-q$ frame is obtained. Assuming a three-phase threewire system, zero-sequence component does not exist, so the $\alpha-\beta$ frame can be obtained from the positive and negative-sequence.

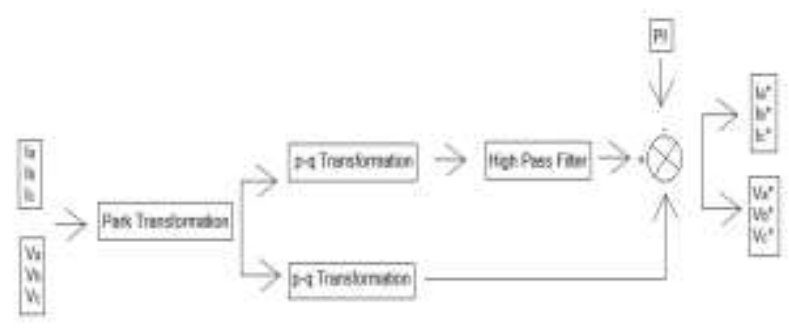

Figure 3 Flow Chart of PQ algorithm

The instantaneous active and reactive power of the nonlinear load is calculated. The AC component is extracted using high pass filter (HPF) to obtain the compensation reference signals in terms of either currents or voltages. The three-phase reference frame is translated to the orthogonal stationary reference frame.

$$
\begin{aligned}
& {\left[\begin{array}{l}
V_{\alpha} \\
V_{\beta}
\end{array}\right]=\sqrt{\frac{2}{3}}\left[\begin{array}{ccc}
1 & -\frac{1}{2} & -\frac{1}{2} \\
0 & \sqrt{\frac{3}{2}} & -\sqrt{\frac{3}{2}}
\end{array}\right]\left[\begin{array}{l}
V_{a} \\
V_{b} \\
V_{c}
\end{array}\right]} \\
& {\left[\begin{array}{l}
I_{\alpha} \\
I_{\beta}
\end{array}\right]=\sqrt{\frac{2}{3}}\left[\begin{array}{ccc}
1 & -\frac{1}{2} & -\frac{1}{2} \\
0 & \sqrt{\frac{3}{2}} & -\sqrt{\frac{3}{2}}
\end{array}\right]\left[\begin{array}{l}
I_{a} \\
I_{b} \\
I_{c}
\end{array}\right]}
\end{aligned}
$$

Instantaneous real power $p$ and reactive power $q$ are given

$$
\left[\begin{array}{l}
p \\
q
\end{array}\right]=\sqrt{\frac{2}{3}}\left[\begin{array}{cc}
V_{\alpha} & V_{\beta} \\
V_{\beta} & -V_{\alpha}
\end{array}\right] \cdot\left[\begin{array}{c}
I_{\alpha} \\
I_{\beta}
\end{array}\right]
$$

From instantaneous reactive power theory, for obtaining the harmonic component, real and reactive power can be decomposed into $\mathrm{DC}(p, q)$ and $\mathrm{AC}$ components (corresponding to the harmonic distortion ) $(p, q)$. From this, the current reference signal is

$$
\left[\begin{array}{c}
I_{a}^{* *} \\
I_{b}^{*} \\
I_{c}^{*}
\end{array}\right]=\sqrt{\frac{2}{3}} \cdot\left[\begin{array}{cc}
1 & 0 \\
-\frac{1}{2} & \frac{\sqrt{3}}{2} \\
-\frac{1}{2} & -\frac{\sqrt{3}}{2}
\end{array}\right] \cdot\left[\begin{array}{cc}
V_{\alpha} & V_{\beta} \\
V_{\beta} & -V_{\beta}
\end{array}\right]^{-1} \cdot\left[\begin{array}{c}
\tilde{p} \\
\tilde{q}
\end{array}\right]
$$

\section{B) RLS algorithm}

Recursive least squares (RLS) is an adaptive filter algorithm that recursively finds the coefficients that minimize a weighted linear least squares cost function relating to the input signals.

Because of its simplicity and improved performance, RLS algorithms are widely used in many applications. Generation of Compensating Current using RLS Algorithm is used in H APF so as to get DC link active current parameter, maintaining DC link voltage with the constant value of each phase and to make ripple free in the waveform.

The value of the instantaneous active current can be obtained from the HAPF in order to compensate for the loss of power and the difference in active power between the load and the supply network. The operation of the RLS algorithm, in which the voltage of the reference 
intermediate circuit and the voltage of the intermediate circuit Vcc (i) have a current sample.

In source voltage, harmonics are not present, so instantaneous voltage given as

$$
V_{x}(t)=V_{a x}(t)
$$

For each phase, the reference active current is given as

$$
i_{a x}(t)=A_{x} V_{x}(t)
$$

The instantaneous reference compensating current specified as

$i(t)=i_{x}(t)-i_{a x}(t)$

RLS algorithms are known for their exceptional performance in variable time environments, but at the cost of increased processing complexity and stability problems. In this algorithm, the filter tap weighting vector is calculated using the $\mathrm{Eq}$

$$
\begin{aligned}
& w(n)=\bar{w}^{T}(n-1)+k(n) \overline{e_{n-1}}(n) \\
& k(n)=u(n) /\left(\lambda+X^{\prime}(n) u(n)\right) \\
& u(n)=\bar{w}_{\lambda}^{-1}(n-1) X(n)
\end{aligned}
$$

intermediate gain vector used to compute tap weights. Where $\lambda$ is a small positive constant very close to, but smaller than 1 .

\section{C) Modified RLS Algorithm}

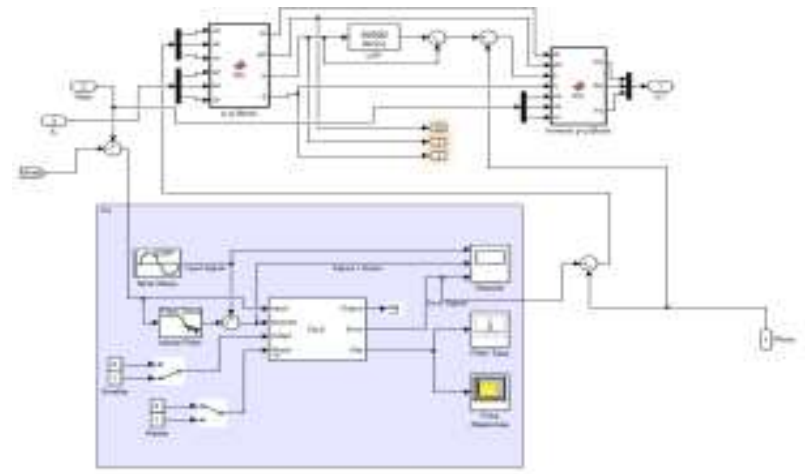

Figure 4 MATLAB/SIMULINK model of proposed algorithm

\section{D) Pulse Generation}

The Pulse Generator block generates square wave pulses at regular intervals. The block's waveform parameters, Amplitude, Pulse Width, Period, and Phase Delay, determine the shape of the output waveform. The following diagram shows how each parameter affects the waveform.

\section{RESULTS AND DISCUSSION}

The analysis of the model was performed in the MATLAB / SIMULINK environment. MATLAB combines easy-to-use iterative analysis and a design desktop environment with a programming language that directly expresses matrix and matrix mathematics. Enables graphical programming to design the system in a simulation environment.

The discrete mode sampling time is kept to be Ts $=5 \times 10^{-}$ ${ }^{6}$. The AC output voltages is then sent to the load. The model can further be integrated with the grid system. In order to overcome the poor and unacceptable performance of the conventional solutions during unsymmetrical loads, an innovative control strategy is anticipated.

- The proposed system presents control strategies of a shunt active power buffer using two algorithms RLS and modified RLS respectively.

- The system is being simulated for 0.5 second for simulation purpose in MATLAB/SIMULINK environment.

- The gate signals provided to the IGBTs drawn after treating the error signals through a control algorithms algorithm.

- The model is made to drive a non linear load model with minimum distortion in the voltage waveform.

The Result analysis of the modern has been arranged in this chapter in the following manner:

1. Case 1: shunt active power filter modelling using rls algorithm only

2. Case2: Hybrid active power filter modelling using modified rls algorithm

3. Load analysis

4. Validation

The table below describes the system parameters of the source that has been feeding the load via power filters.

\begin{tabular}{|l|l|}
\hline \multicolumn{2}{|c|}{ Table 5.1 : System Parameters: } \\
\hline \multicolumn{2}{|c|}{ Voltage Source Inverter } \\
\hline $\begin{array}{l}\text { Phase to phase voltage } \\
\text { (Volts) }\end{array}$ & 400 \\
\hline Frequency (Hertz) & 50 \\
\hline Source resistance (ohms) & 0.001 \\
\hline Source Inductance (H) & $1 \times 10^{-8}$ \\
\hline \multicolumn{2}{|c|}{ Power Filter } \\
\hline
\end{tabular}




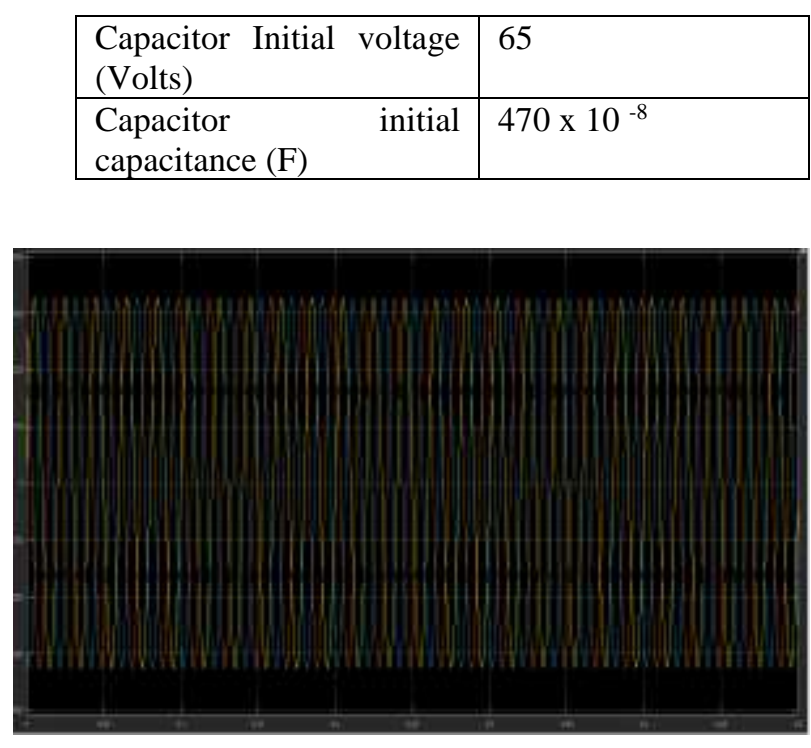

Figure 5 Source Voltage Output From the three phase voltage source

CASE 1: Model Using RLS algorithm only.

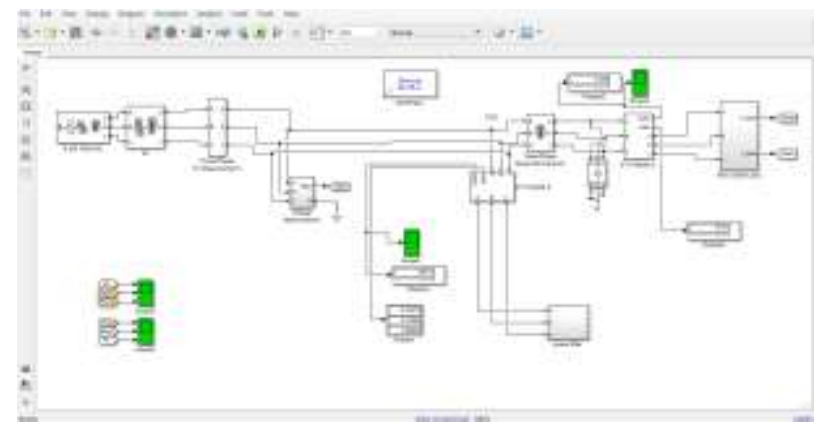

Figure 6 MATLAB Simulink Model of Shunt Active Power filter with simple RLS algorithm

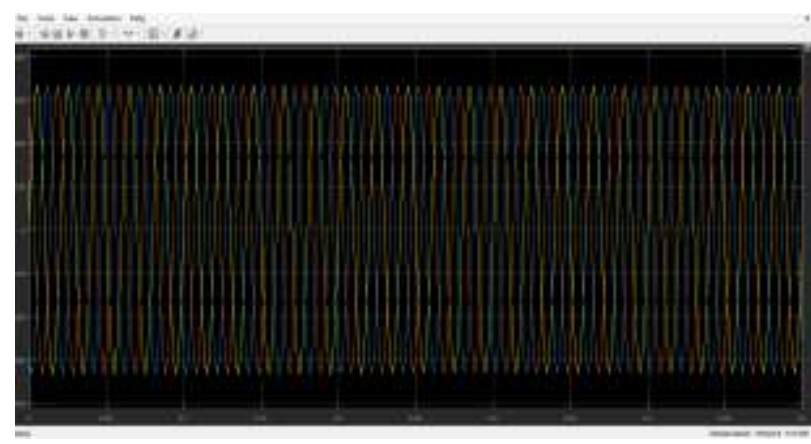

Figure 7: Input voltage to the load

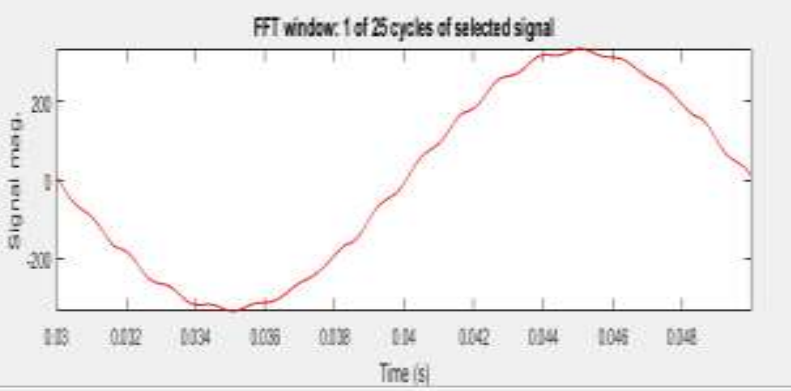

Figure 8: FFT analysis of Input voltage to the nonlinear load

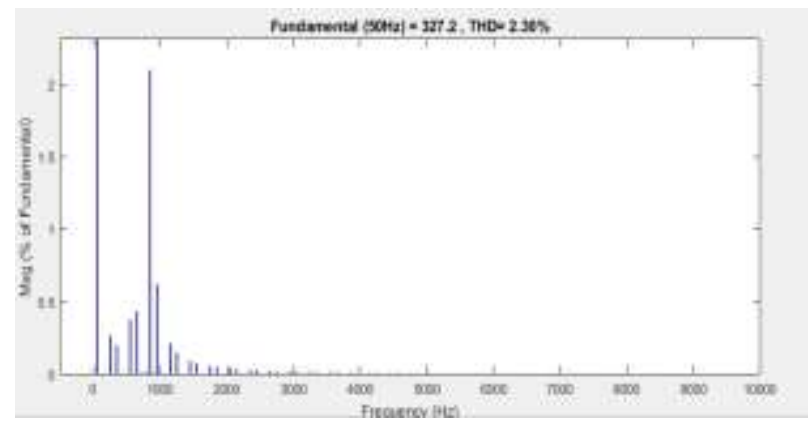

Figure $9 \mathrm{THD}=2.30 \%$ in Input voltage to the nonlinear load

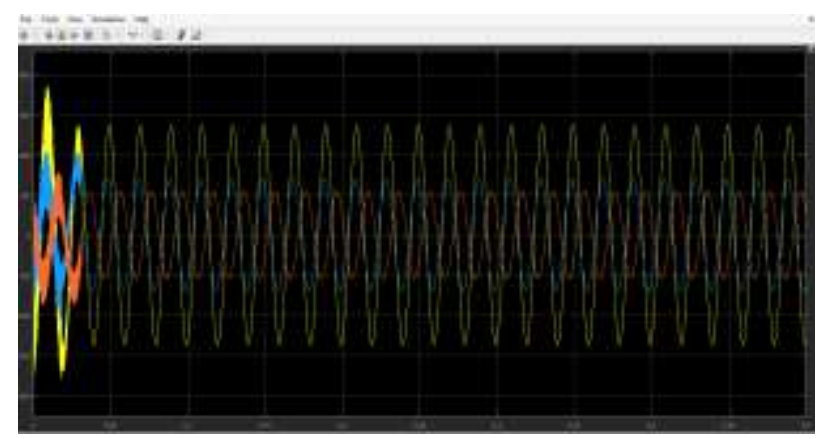

Figure 10: Output voltage from the power filter

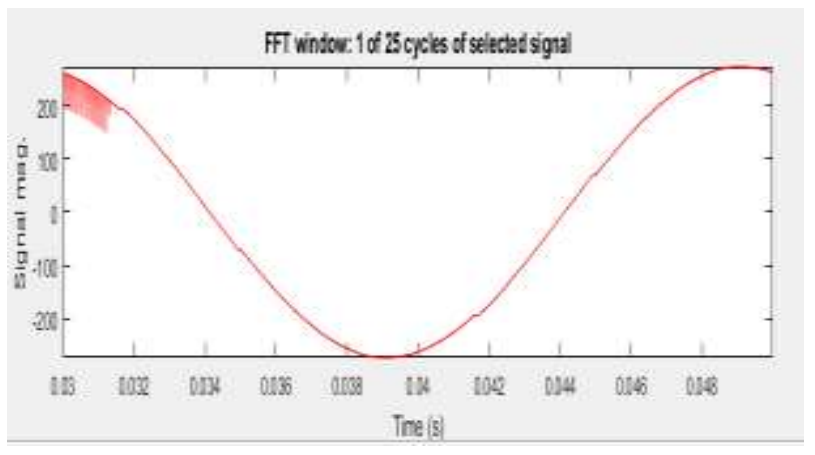

Figure 11: FFT analysis of Output voltage from the power buffer 


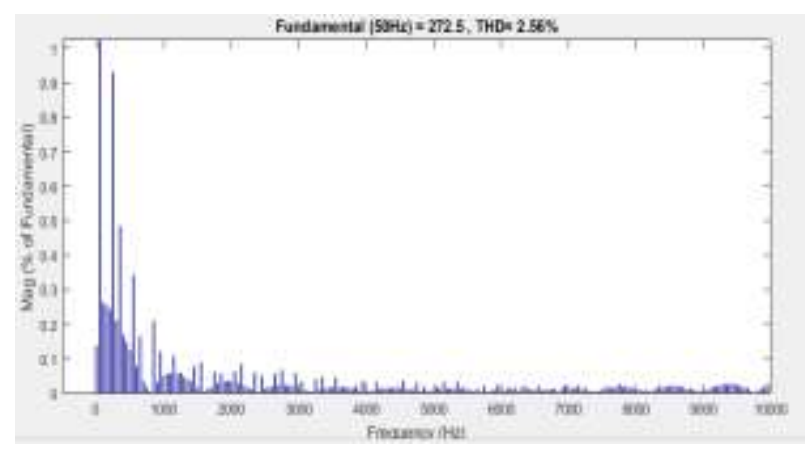

Figure 12: $\mathrm{THD}=2.56 \%$ Output voltage from the power buffer

\section{A) Model Using Modified RLS algorithm in HAPF}

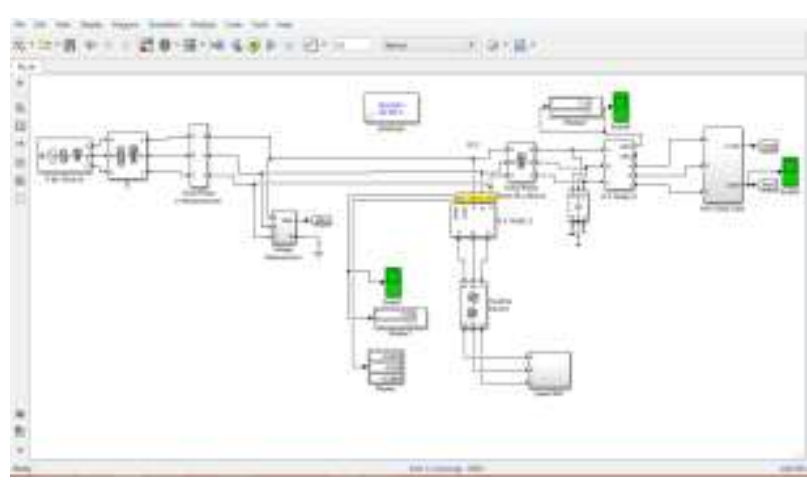

Figure 13: MATLAB Simulink Model of Hybrid Active Power filter with modified RLS algorithm

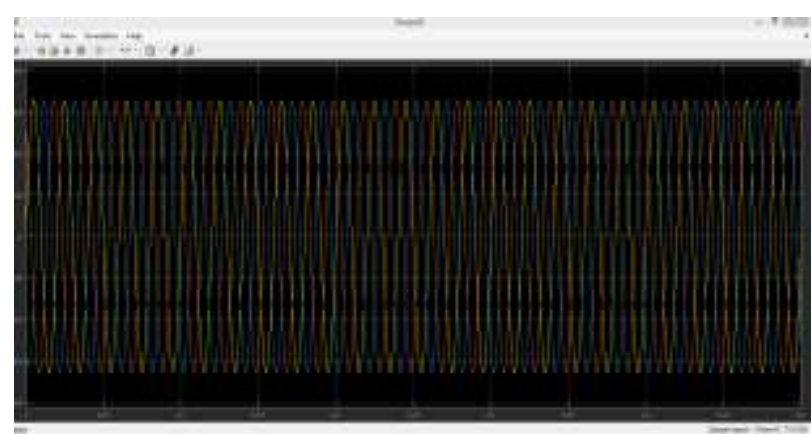

Figure 14 Input voltage to the load

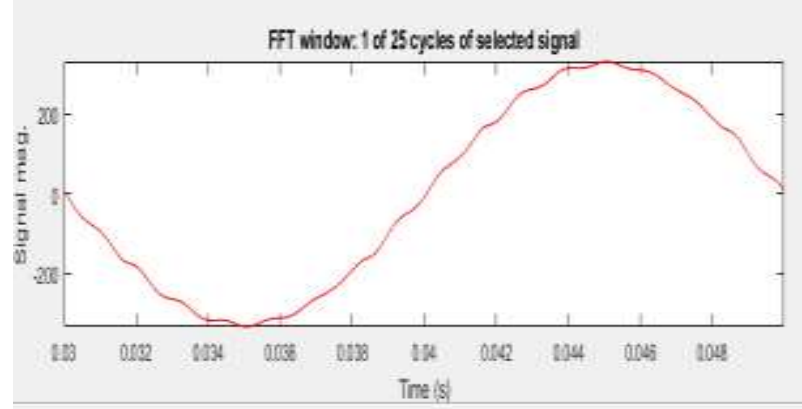

Figure 15 FFT analysis of Input voltage to the nonlinear load

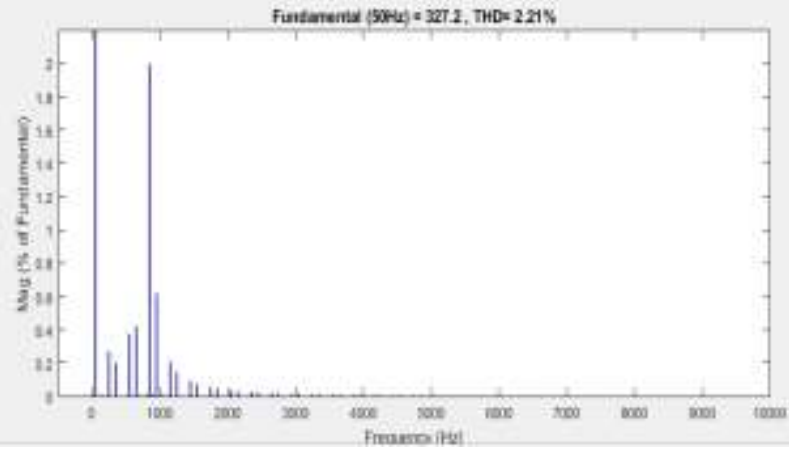

Figure $16 \mathrm{THD}=2.21 \%$ in Input voltage to the nonlinear load

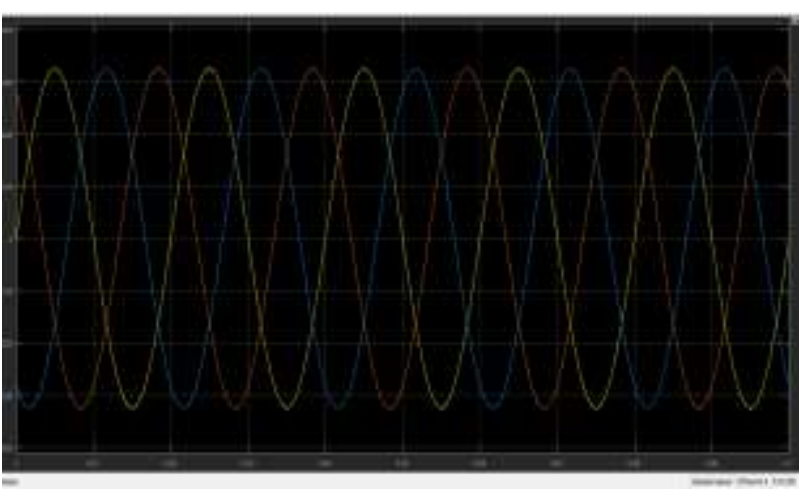

Figure 17 Output voltage from the power filter

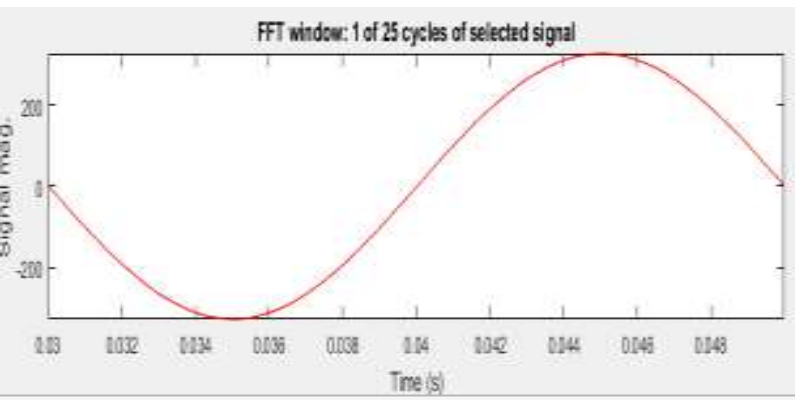

Figure 18 FFT analysis of Output voltage from the power buffer

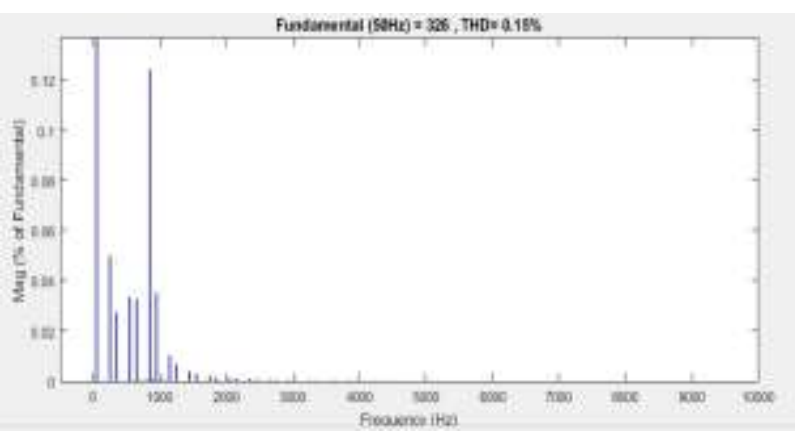

Figure $19 \mathrm{THD}=0.15 \%$ in Output voltage from the power buffer

B) Analysis of models at linear and nonlinear load 


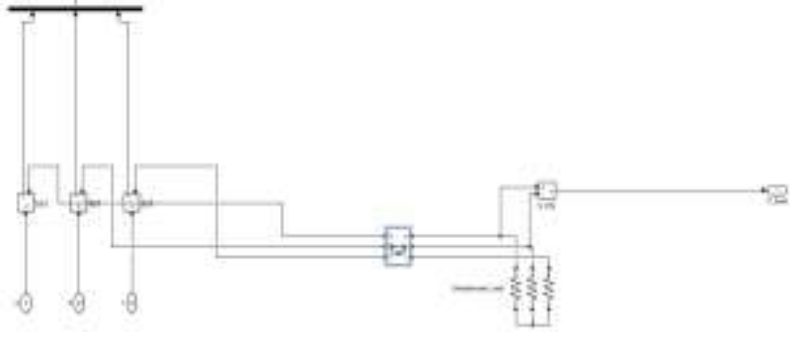

Figure 20 Modeled Unbalanced/ Non Linear Load in MATLAB

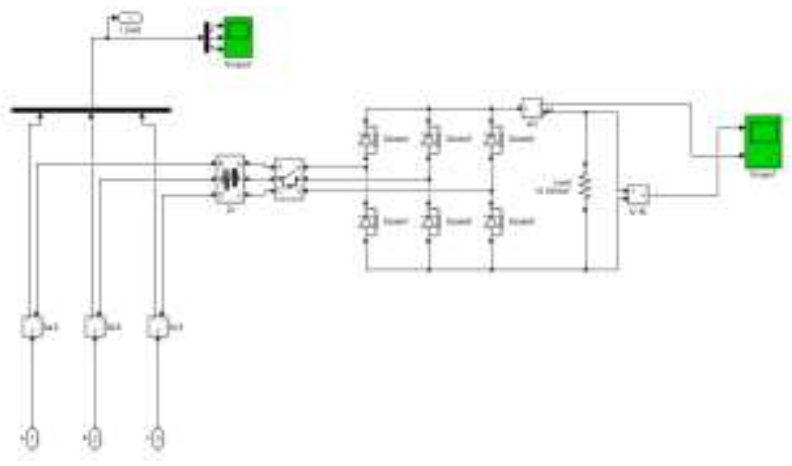

Figure 21 Modeled Linear load in MATLAB

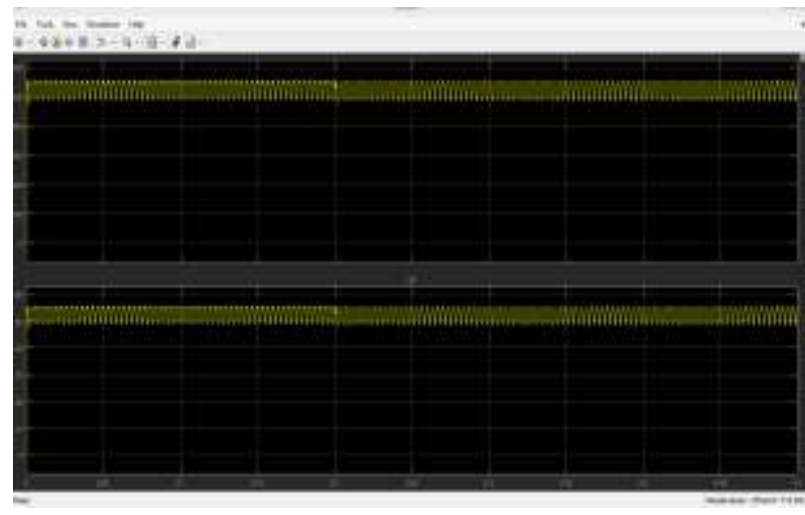

Figure 22 Current and Voltage outputs at the linear load terminal

\section{D) Validation}

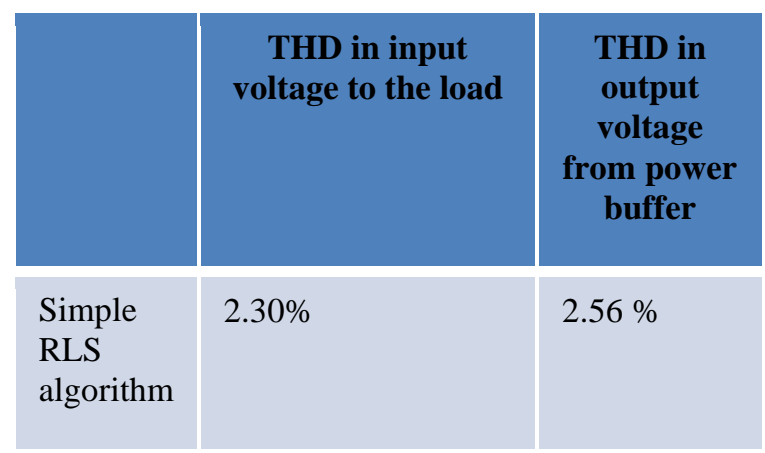
Proposed
$2.21 \%$
$0.15 \%$
Modified
RLS
algorithm

The THD analysis of two waveforms has been done in this work. One at the voltage input to the nonlinear load and other of the voltage output from the power buffer after using two types of algorithms. This concludes that the proposed algorithm has been proved more effective in reducing the distortion level

\section{CONCLUSION}

As technology advances, the demand for exponential electricity increases. Many consumer devices constantly demand quality performance for their operation. The performance of the devices depends to a large extent on the quality of the power supply. However, the quality of the end user's energy is influenced by various external and internal factors. They are like fluctuations of voltage and frequency, faults, faults, etc. These energy quality problems reduce the life and efficiency of the equipment. Therefore, these problems should be mitigated to improve the performance of the consumer equipment and the overall performance of the system.

In this work a HAPF has been designed using modified RLS algorithm with an objective to reduce the Total Harmonic Distortion in the voltage output waveforms. The following major points we concluded from the above analysis:

The total harmonic distortion in the voltage output waveform being fet to the load using only rls algorithm is found to be $2.30 \%$ and the THD using proposed modified rls algorithm which is being merged with $\mathrm{P}$ and $\mathrm{Q}$ algorithm is found to be $2.21 \%$.

In case of the output voltage from the power buffer using RLS algorithm only the THD level is $2.56 \%$ and using modified rls algorithm the THD is $0.15 \%$.

The distortion level in the output voltage waveforms in both the cases being fed to the load when compared, it is found that rls algorithm in combination with PQ algorithm is more effective in reducing the distortion as compared to standard rls method.

The distortion level has been considerably reduced in order to get a smooth voltage waveform that would be able to drive the load, both linear and nonlinear more effectively and efficiently 
Energy quality can be improved by using a more efficient power filter and algorithm in a networked system. The work can be expanded to improve the quality of the network powered by renewable energy sources. By improving the signal quality of the output voltage, it can be further integrated into the network. The modulation technique is simple and easy to implement. Using real facts can make the UPS more robust and easier. With the advent of more powerful DPS, the computational complexity and the memory usage needs of the algorithms will decrease and more sophisticated and efficient algorithms can be implemented. It is therefore certainly true that the field of adaptive filtering will remain a longopen field for scientific research and commercial applications.

\section{REFERENCES}

[1] Vijanth S. Asirvadam, M.M. Hussam. Alhaj "Comparison of Power System Harmonic Prediction” DOI: 10.1016/j.protcy.2013.12.238

[2] M. Kelnar, Radek Martinek "Control Methods of Active Power Flters using Soft Computing Techniques" September 2015

[3] ChamandeepKaur "power quality improvement using hybrid filter" Volume No 03, Special Issue No. 01, March 2015

[4] V.K. Arun Shankar, N. Senthil Kumar, "Implementation of Shunt Active Filter for Harmonic Compensation in a 3 Phase 3 Wire Distribution Network", Energy Procedia Volume 117, June 2017, Pages 172-179. 